\title{
Diametrical User Control to Filter the Unwanted Messages from Online Social Networks
}

\author{
M. Swapna \\ Department of CSE \\ M. tech student MITS, \\ Madanapalle.
}

\author{
P.Rajarajeswari \\ Department of CSE \\ Assoc. Professor \\ MITS, Madanapalle
}

\author{
D.Vasumathi, Ph.D \\ Professor \\ JNTU, Hyderabad
}

\begin{abstract}
Online Social Networks (OSNs) are the most impulsive standard to provide communication, sharing and disseminate a significant amount of individual life data. Content can be changed based on daily needs in several communication fields (audio, video, image, and text). One of the important aspects of OSN is to give the user capacity to automatically manage the messages posted and also to filter the unnecessary messages. The main aim of my work is to evaluate an automated system called Filter walls and also using Machine learning text categorization technique. These techniques automatically label the messages in support of substancebased filtering. This application focuses on Face book where unknown persons post unwanted comments for the uploaded photos. So, our system provides help and security to our profiles by directly controlling what kind of messages should be posted on our own walls.
\end{abstract}

\section{INTRODUCTION}

Now a days Online Social Networks (OSNs) are one of the most trendy interactive way to interact, sharing, and to disseminate the most significant amount of individual life. Day to day continuous interactions involve the conversation of various types of data, including free text, image, audio, and video data. In the sharing of OSN data filtering can also be used for a different, more sensitive, purpose. This is due to the fact that in OSNs there is a possibility of writing or commenting other posts on particular areas (private/public). Information filtering gives the users directly manage the unwanted messages.

\section{EXISTING SYSTEM}

The Users cannot control the messages on their public wall. Existing system may not secure because after completion of the classification, it posted the message. Existing system has to use content based classifiers. The classifier used to filter the unwanted messages. Previously defind some techniques are web content mining techniques using different application

\section{PROPOSED SYSTEM}

Proposed system gives the control to what kind of messages posted on own walls. It is secure because the filter wall acts as administrator. The core components of the proposed system are the content based and the short text classifier modules. Here we used rule layer for filtering the unwanted messages .We start by recounting the use of blocked lists.

\section{SYSTEM ARCHITECTURE}

The OSN have three layers, there are graphical user interface, social network application and social network managers. The social network managers handle the

Application layer have short text classifier and content based message filtering. Short text classifier classifying the messages based on the content. Content based message filter have black list and filtering policies. First, find relationship between the user and message senders and it will filter and calculate the probabilities using classifier. And the send a empty message below the probabilities result to the user. So our proposed system will give the direct control to the user that what kind of messages displays on their wall.

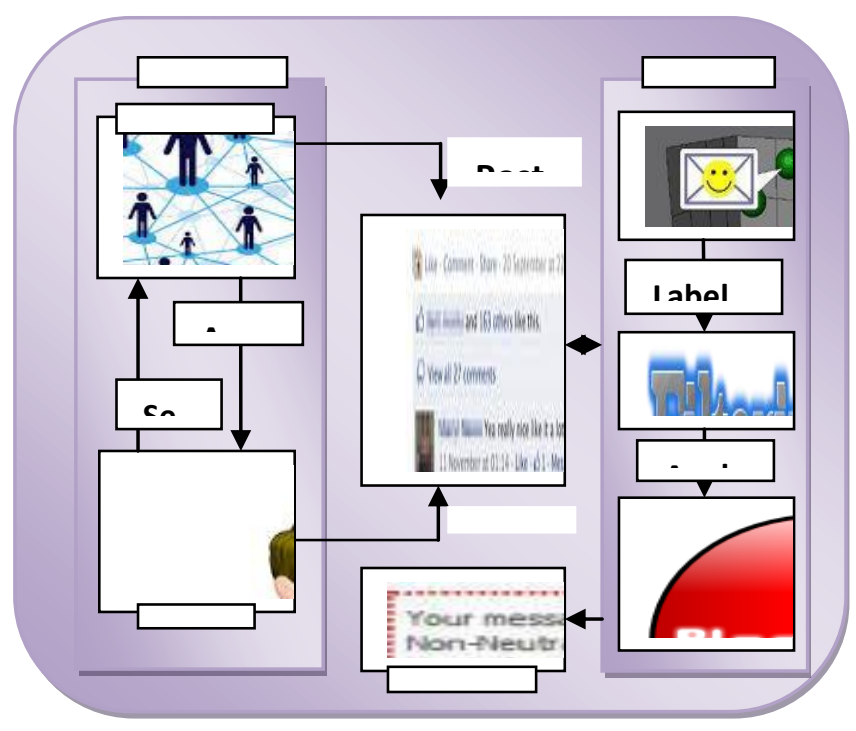

\section{ALGORITHM}

\subsection{Machine Learning (ML) Text Categorization Techniques}

Step 1: Short Text Classifier

i. Text Representation

The text representation using three types of features first one is bag of word (BOW), second one is Document properties (dp),

And third Contextual Features. the resulting information is limited within the text of the message. However, the operational environment is acceptable to use knowledge, if any supply of information that is outside the message body but any way it is related to the message itself. 


\section{ii. Machine Learning-Based Classification}

The short text classification is a two level classification process. The first-level classifier performs a binary hard classification that labels messages as Neutral and Non-neutral. Second-level assignment in which a fine-grained classification is performed.

\section{iii. Radial Basis Function Networks (RBFN)}

RFBNs is a protocol it is a processing unit with local activation domain here using Gaussian function is mainly introduced as a nutral network evalution of approximation..

\section{Step 2: Filtering Rules and Blacklist Management}

\section{i. Filtering Rules}

\section{Creator specification}

It requires to state conditions on type, depth, and trusted values of the relationship(s).Creators should be involved in order to apply some specified rules. The creator specification (creatorSpec) is perfectly denotes a set of OSN users

- A set of attribute constraints in the form of operation by the user profile name, value and operation.compatible with an's domain.

- A set of relationship constraints of the form (m, rt, minDepth, max Trust) denoting all the OSN users participating with user $\mathrm{m}$ in a relationship of type $\mathrm{RT}$, having a depth greater than or equal to min Depth, and a trust value less than or equal to max Trust.

\section{CONCLUSION}

We have presented system direct control to the user block unwanted messages on their social network wall. The system using the machine language soft classifier to label the contents is Neutral and Nonneutral. And then applying the Filter Rule based on the creators.

\section{REFERENCES}

[1] M. Chau and H. Chen, "A Machine Learning Approach to Web Page Filtering Using Content and Structure
Analysis," Decision Support Systems, vol. 44, no. 2, pp. 482-494, 2008.

[2] R.J. Mooney and L. Roy, "Content-Based Book Recommending Using Learning for Text Categorization," Proc. Fifth ACM Conf. Digital Libraries, pp. 195-204, 2000.

[3] F. Sebastiani, "Machine Learning in Automated Text Categorization," ACM Computing Surveys, vol. 34, no. 1, pp. 1-47, 2002

[4] M. Vanetti, E. Binaghi, B. Carminati, M. Carullo, and E. Ferrari, "Content-Based Filtering in On-Line Social Networks," Proc. ECML/PKDD Workshop Privacy and Security Issues in Data Mining and Machine Learning (PSDML '10), 2010.

[5] N.J. Belkin and W.B. Croft, "Information Filtering and Information Retrieval: Two Sides of the Same Coin?" Comm. ACM, vol. 35, no. 12, pp. 29-38, 1992.

[6] P.J. Denning, "Electronic Junk," Comm. ACM, vol. 25, no. 3, pp. 163-165, 1982.

[7] P.W. Foltz and S.T. Dumais, "Personalized Information Delivery: An Analysis of Information Filtering Methods," Comm. ACM, vol. 35, no. 12, pp. 51-60, 1992. [9] P.S. Jacobs and L.F. Rau, "Scisor: Extracting Information from On- Line News," Comm. ACM, vol. 33, no. 11, pp. 88 97, 1990.

[8] S. Pollock, "A Rule-Based Message Filtering System," ACM Trans. Office Information Systems, vol. 6, no. 3, pp. 232-254, 1988.

[9] P.E. Baclace, "Competitive Agents for Information Filtering," Comm. ACM, vol. 35, no. 12, p. 50, 1992.

[10] M.J. Pazzani and D. Billsus, "Learning and Revising User Profiles: The Identification of Interesting Web Sites," Machine Learning, vol. 27, no. 3, pp. 313-331, 1997. 